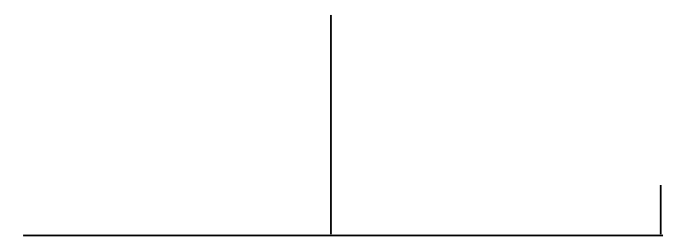

Rev. Latinoam. Psicopat. Fund., São Paulo, v. 15, n. 2, p. 293-308, junho 2012

\title{
Considerações sobre o diagnóstico precoce na clínica do autismo e das psicoses infantis
}

Este artigo se refere a uma pesquisa de Iniciação Científica. Esta se baseou na teoria de Marie-Christine Laznik para investigar, por meio do estudo de prontuários, como aconteceu em um Centro de Atenção Psicossocial Infantil, localizado no município de São Paulo, o diagnóstico precoce do autismo e das psicoses infantis e como se deu a trajetória destas famílias até a chegada à instituição. A pesquisa revelou que o início do tratamento de crianças autistas e psicóticas se dá de maneira tardia em consequência da não realização da detecção precoce, da demora por parte de profissionais elou instituições de saúde em dar um diagnóstico formal e realizar um encaminhamento e da insegurança dos profissionais da saúde para lidar com estas psicopatologias graves. Os resultados desse trabalho ajudam a pensar ações futuras e necessidades de maior concentração de esforços para que o tratamento do autismo e das psicoses infantis possa acontecer de maneira cada vez mais precoce, frente a quadros não cronificados.

Palavras-chave: Autismo infantil, criança, diagnóstico precoce, psicoses, saúde mental 


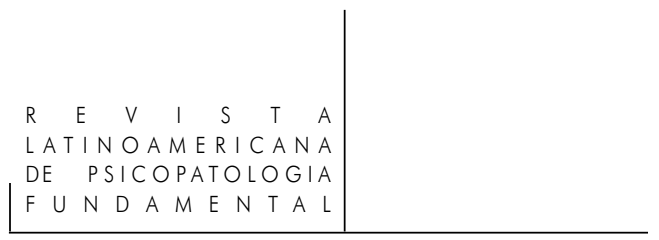

\section{Introdução}

\section{A pesquisa}

Este artigo se refere a uma pesquisa de Iniciação Científica Captação de bebês de risco: detecção de psicopatologias graves. Financiada pelo Programa Institucional de Bolsas de Iniciação Científica - Conselho de Ensino Pesquisa e Extensão, a pesquisa se estendeu por 12 meses e teve um Centro de Atenção Psicossocial Infantil, localizado no município de São Paulo, como instituição parceira. Por outro lado, está inserida em um grupo de pesquisa vinculado ao Projeto espaço palavra da clínica psicológica Ana Maria Poppovic (PUC-SP), o qual oferece serviço psicológico para crianças e adolescentes com organizações subjetivas como o autismo e a psicose.

A necessidade da pesquisa surgiu da constatação, por parte dos responsáveis pelo Projeto espaço palavra, de uma dificuldade, experimentada por profissionais e instituições de saúde, em efetuar prontamente, o diagnóstico dessas psicopatologias. Em consequência, as crianças autistas e psicóticas chegam ao Projeto com um histórico preenchido pela passagem por profissionais e/ou instituições de saúde em busca de uma resposta diagnóstica e de um tratamento.

Para viabilizar a intervenção precoce - ou seja, anterior à cronificação do quadro autístico ou psicótico - é pressuposto básico minimizar os descaminhos até o atendimento. Assim, esta pesquisa consistiu em investigar, norteada pela teoria da psicanalista Marie-Christine Laznik, os descaminhos trilhados pelas famílias de crianças atendidas como autistas ou psicóticas por um Centro de Atenção Psicossocial Infantil.

\section{O problema de pesquisa}

O autismo é uma psicopatologia grave proveniente da não instauração sucessiva de estruturas psíquicas fundamentais, diante da 
qual existe o risco de instalação de deficiências irreversíveis para o desenvolvimento psíquico da criança. Nos termos da psicanalista Marie-Christine Laznik (2004), isso se revelaria na ausência do olhar, na não inscrição do outro, na ausência do terceiro tempo do circuito pulsional e na falta da representação dos representantes, ou seja, da formação de redes entre as inscrições psíquicas. A partir dessa definição, Laznik constrói sua proposta clínica baseada numa intervenção no laço Outro primordial/criança, isto porque considera o Outro como fundador do aparelho psíquico.

Com base nessa mesma definição, o autismo exige um diagnóstico e uma intervenção o mais rápido possível, única maneira de reduzir a probabilidade de cronificação. A intervenção precoce no quadro de autismo não só aumenta as possibilidades de tratamento, como ainda minimiza alguns sintomas experimentados pelos pais, agravados com o passar do tempo (Laznik, 1997): depressão, perda da capacidade tanto de supor um sujeito na criança quanto de revelar-se como faltante, e, por fim, a impossibilidade de impor "nãos" a esta criança.

Segundo Laznik (2000):

Sabemos que provavelmente há uma "psicossomática" do autismo, isto é, que o não uso psíquico do aparelho neuronal vai conseguir lesá-lo. A hipótese, mais ou menos implícita, que sustenta esta investida em direção ao precocíssimo, é que haveriam (sic) meios de recolocar em funcionamento estruturas em vias de constituição. [...] Diante desta patologia, luta-se contra o relógio. (p. 76)

Para realizar a detecção precoce do autismo, Laznik (2000) propõe o uso de "sinais de detecção precoce", ou seja, de "elementos clínicos que fazem parte de um conjunto metapsicológico coerente, relacionado com as condições de constituição de todo sujeito humano" (p. 78). Tais sinais indicam falhas no desenvolvimento de certas estruturas, necessárias à organização do aparelho psíquico e, consequentemente, à constituição de um sujeito.

Assim, esses sinais deveriam ser traduzidos em "fatos de observação clínica" a serem ensinados aos médicos da primeira infância, isto porque a parceria com esses profissionais é indispensável no trabalho de detecção precoce do autismo e das psicoses infantis: na maioria das vezes, eles, e não os psicólogos, têm acesso a essas crianças nos primeiros anos de vida. Porém, devemos pensar a pesquisa e a clínica do autismo em uma época na qual já existe, na pedopsiquiatria europeia, a preocupação com o diagnóstico precoce. Apesar disso, como nos ensina Laznik (2000), os "meios médicos, principalmente hospitalares-universitários, têm pouco ou nenhum contato" (p. 76) com a obra de Lacan, o que pode resultar no desconhecimento, não só da importância do diagnóstico precoce como, ainda, dos meios de colocá-lo em prática em suas consultas. 
Cabe aos psicólogos, por meio da difusão de conhecimento, contribuir no sentido de tornar o encontro de médicos da primeira infância com duplas mãe/ bebê também um espaço para a avaliação do laço Outro primordial/bebê, de modo que os mesmos possam "imaginar que nem tudo vai necessariamente bem num lactante, mesmo se suas funções biológicas são ritmadas como convém" (Idem, p. 79)

Dois sinais foram, então, caracterizados pela autora.

O primeiro consiste no não olhar entre o Outro primordial e a criança, onde o olhar deve ser entendido, não como visão, mas sim como sinônimo de atenção, de investimento. Quando um bebê olha no rosto de sua mãe, este funciona como um espelho, ele vê a si mesmo. Porém, se o rosto da mãe somente refletir a rigidez de suas defesas, o bebê o evitará a todo custo. Como o olhar estruturante do Outro é base para o estádio do espelho, este não ocorrerá da maneira esperada na ausência do outro, ficará comprometido então o sentimento de unidade corporal do bebê. Ficará comprometida, ainda, a constituição do narcisismo primário, advindo do olhar deste mesmo Outro.

O segundo sinal - a ser investigado somente nos bebês cuja existência do primeiro foi confirmada - consiste na não instauração do circuito pulsional completo. No caso do autismo, o terceiro tempo pulsional não acontece e, como consequência, o circuito pulsional não pode se completar (Laznik, 2004).

De acordo com a autora, quando não existe o terceiro tempo pulsional não se pode ter certeza do caráter pulsional dos outros dois tempos: "nada garante que o autoerotismo não fique desprovido de eros [...], nada garante que o polo alucinatório de satisfação esteja no circuito e que, portanto, todo o sistema de representações possa funcionar" (p. 146).

Lidar com o autismo se mostra uma árdua tarefa para os pais. Segundo Laznik, num quadro de autismo, muitas vezes é impossível, para os pais, realizar funções básicas do Outro primordial, indispensáveis para o sujeito advir. Essa impotência toma as figuras parentais quando suas tentativas e investimentos em direção à criança autista são fracassados. Quando o bebê não responde, a mãe é colocada em uma situação na qual o filho parece ausente, torna-se impossível a ocorrência da prosódia materna, do chiste e da protoconversação, resultantes da relação mãe/bebê e, portanto, inexistentes na ausência de uma das partes. De acordo com Laznik et al. (2006, p.104), uma mãe só conseguirá assumir sua função de Outro primordial - a loucura necessária proposta por Winnicott como indispensável para a constituição do sujeito - nas "condições de segurança da capacidade materna".

Por isso mesmo, cabe ao analista, em um primeiro momento - no qual as figurais parentais não conseguem exercer a função de Outro primordial - oferecer uma escuta analítica frente às produções sonoras da criança, por mais sem sentido 


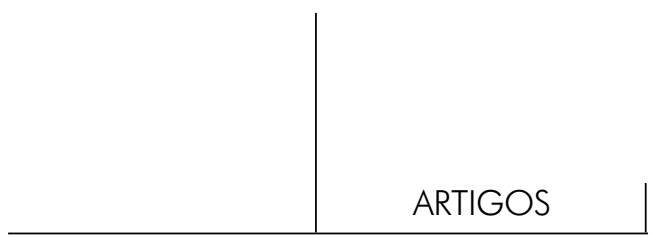

que possam parecer; e assim, dar conta da ilusão antecipatória e ocupar a posição de Outro faltante. Somente a partir da realização dessas funções a criança terá aberta a possibilidade de advir como sujeito desejante. Além disso, ao assumir o papel de Outro primordial frente a essa criança, o analista abre para os pais a possibilidade de a olharem como um ser digno em sua luta para se tornar um sujeito, e colabora, assim, para a construção da segurança na realização das funções parentais. A intervenção do analista frente à própria criança e aos seus pais se faz tão mais significativa quanto mais precoce for o início do tratamento (Laznik, 1997).

Apesar da possibilidade e mesmo da necessidade de intervenção no quadro autístico antes de sua cronificação, o diagnóstico precoce foi identificado como gerador de dúvidas e insegurança, por apresentar uma sintomatologia difusa e manifestações clínicas sutis, em seu estado inicial.

Objetivos gerais e específicos

Com base na relevância do diagnóstico precoce e do início de um tratamento anterior à cronificação do quadro de uma psicopatologia grave, esta pesquisa investigou o caso específico do autismo. E teve como objetivos gerais investigar, por meio da análise de prontuários de pacientes atendidos em um Centro de Atenção Psicossocial Infantil, a trajetória e os descaminhos vividos pelas famílias de crianças com psicopatologias graves, e assim esclarecer as condições nas quais foi (ou não) possível efetuar diagnósticos precoces.

Como objetivos específicos, a pesquisa pretendeu traçar um contraste entre os perfis da trajetória dos pacientes diagnosticados por esta instituição como autistas e como psicóticos. Ao final dos trabalhos, ocorreu uma devolutiva dos resultados obtidos para os profissionais do Centro de Atenção Psicossocial Infantil, de modo a colaborar com a instituição parceira para uma reflexão a respeito de suas práticas clínicas. Assim, a investigação teve o intuito de colaborar para a redução dos obstáculos ao acesso precoce da criança a um atendimento às patologias em questão.

\section{Metodologia}

Como já mencionado, a pesquisa consistiu, basicamente, na investigação dos prontuários de crianças diagnosticadas como autistas e psicóticas por um Centro de Atenção Psicossocial Infantil. Prontuários são documentos únicos para cada paciente. Nele, se encontram cópias de documentos pessoais, informações 
referentes à situação socioeconômica e a constituição familiar do paciente, os termos de encaminhamento para a instituição e a entrevista inicial com os pais ou responsáveis pela criança. Além disso, quando tem início o tratamento, o prontuário é utilizado pelos profissionais para o registro dos atendimentos, tanto da criança quanto dos pais ou responsáveis.

\section{Procedimentos de coleta e análise de dados}

Os prontuários foram selecionados pela Diretora da instituição parceira de acordo com a necessidade da pesquisadora em estudar prontuários de crianças diagnosticadas com autismo ou psicose. Não foram utilizados prontuários de pacientes cujo tratamento já havia sido encerrado durante o período da vigência da pesquisa, uma vez que esses casos, em princípio, já teriam recebido um tratamento bem sucedido. Assim, não se justificaria, do ponto de vista ético, restabelecer um vínculo desses sujeitos com a instituição em questão.

\section{Análise e organização dos dados}

O estudo dos prontuários selecionados se deu em meio a visitas semanais ao Centro de Atenção Psicossocial Infantil. Durante o período da vigência da pesquisa, havia aproximadamente 200 pacientes em atendimento na instituição. Foram analisados 34 prontuários, sendo 14 de pacientes autistas e 20 de pacientes psicóticos, perfazendo-se, assim, o total de prontuários disponíveis relativos a pacientes autistas ou psicóticos em tratamento.

A análise das informações contidas nos prontuários teve como foco a trajetória realizada por estas famílias antes da chegada à instituição. Por isso mesmo, o tratamento recebido pelos pacientes no Centro de Atenção Psicossocial Infantil não foi objeto de pesquisa.

A partir dos prontuários, algumas informações foram selecionadas: número do prontuário, nome do paciente, data de nascimento, nome da mãe, composição da família, histórico do paciente, origem do encaminhamento ao Centro de Atenção Psicossocial Infantil, data de chegada e diagnóstico. Estas informações se revelaram importantes, tanto para a realização da pesquisa, no caso da necessidade de algum esclarecimento, quanto para possibilitar a outros pesquisadores a utilização dos mesmos dados.

Terminada a etapa de coleta de informações, essas foram interpretadas, com o objetivo de selecionar as informações relevantes para a pesquisa e, assim, obter descritores capazes de caracterizar e diferenciar cada uma das trajetórias em questão. 


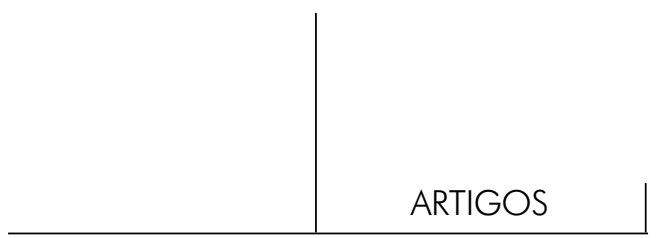

Como resultado deste trabalho, foram criados 13 descritores:

1) Idade na qual os pais percebem, pela primeira vez, sinais de alguma coisa de errado com seus filhos, tornando-se objeto de preocupação.

2) Sinais precoces percebidos pela mãe durante a anamnese feita em sua primeira visita ao Centro de Atenção Psicossocial Infantil.

3) Idade na qual profissionais ou instituições da saúde ${ }^{1}$ diagnosticaram essas crianças como autistas ou psicóticas.

4) Tempo transcorrido entre a percepção dos pais, de algo errado com seu filho, e o primeiro diagnóstico de autismo ou psicose estabelecido por algum profissional ou instituição de saúde.

5) Idade de início do primeiro tratamento.

6) Idade de início do tratamento no Centro de Atenção Psicossocial Infantil.

7) Tempo transcorrido entre o primeiro atendimento pelo profissional ou pela instituição consultada até o início de seu tratamento no Centro de Atenção Psicossocial Infantil.

8) Número de profissionais ou instituições procurados antes do diagnóstico formal. $^{2}$

9) Número de crianças encaminhadas ao Centro de Atenção Psicossocial Infantil por instituições ou profissionais da saúde e por escolas.

10) Primeiro lugar de tratamento específico para o diagnóstico de autismo ou psicose.

11) Diagnósticos não esclarecidos.

12) Existência de outros casos ligados à saúde mental na família do paciente.

13) Relação do diagnóstico da criança com a vivência em uma família desestruturada. ${ }^{3}$

\section{Resultados e discussão}

Analisando-se os prontuários à luz desses descritores, foi possível elaborar uma tabela contrastiva entre pacientes com diagnóstico de autismo e de psicose.

1. Neste trabalho, são consideradas instituições e profissionais de saúde autônomos separadamente.

2. Pacientes que receberam um diagnóstico formal já na primeira instituição a que recorreram entram na faixa de 1 profissionais/instituições procurados.

3. Famílias desestruturadas são entendidas aqui como aquelas permeadas por violência, drogadição, separações frequentes dos cônjuges etc., sendo assim, ambientes com elementos potencialmente nocivos à saúde mental.

Rev. Latinoam. Psicopat. Fund., São Paulo, v. 15, n. 2, p. 293-308, junho 2012 
A tabela, apresentada a seguir, organiza-se em tornos dos 13 descritores discutidos na seção anterior deste artigo. Cada descritor foi organizado separadamente, por meio de dois padrões distintos.

Tabela 1: Dados contrastados entre pacientes diagnosticados como autistas e psicóticos em tabelas formuladas por descritores.

Quando se trata do autismo e da psicose infantil, os descaminhos apresentados como frequentes pelos profissionais do Espaço palavra, tornam-se barreiras para as possibilidades de desenvolvimento de uma criança.

Os dados obtidos no Centro de Atenção Psicossocial Infantil estudado evidenciam o início de um tratamento, para as crianças diagnosticadas com autismo e psicose infantil, mais tarde do que o esperado. Isso se dá, basicamente, por três motivos: a não realização da detecção precoce; a demora por parte de instituições e/ou profissionais de saúde em estabelecer o diagnóstico e realizar um encaminhamento; a insegurança na realização de um tratamento adequado à patologia por parte de profissionais e instituições de saúde.

Em 78,6\% dos casos de crianças autistas, os pais já haviam percebido algo de errado com seus filhos antes de um diagnóstico formal por parte de algum profissional ou instituição de saúde. Nesses casos, sinais foram percebidos, em $36,4 \%$ dos casos, quando a criança tinha um ano ou menos; em $27,3 \%$, aos dois anos; e em 18,2\% dos casos, quando a criança tinha três anos.

Já no que diz respeito às psicoses, em $45 \%$ dos casos os pais já haviam percebido que havia algo de errado com seus filhos antes de um diagnóstico formal. Nesse grupo, sinais foram percebidos em $11,1 \%$ dos casos quando a criança tinha dois anos; em $22,35 \%$, quando a criança tinha quatro anos; em $22,35 \%$, aos sete anos; em 33,3\% dos casos, com oito anos ou mais.

As principais razões desses pais imaginarem algo de errado com seus filhos envolvem, entre outros motivos, sinais de detecção precoce de bebês com risco de se tornarem autistas ou psicóticos. No caso do autismo, por exemplo, em $63,6 \%$ dos casos a principal razão que levou os pais a imaginarem algo de errado com seus filhos foi a ausência de linguagem.

Apesar dessa percepção parental revelar-se precoce, principalmente no caso do autismo, o diagnóstico formal e o consequente início de um tratamento não se dão de imediato.

O período transcorrido entre essa percepção dos pais e o início de um tratamento é preenchido por passagens por profissionais e instituições de saúde. Em casos mais graves, os pacientes passam por até seis profissionais e/ou instituições de saúde, consequentemente, iniciando o tratamento em idades mais avançadas. 


\section{ARTIGOS}

Em relação ao autismo, em 14,3\% dos casos se passou menos de um ano entre a percepção inicial dos pais e o primeiro diagnóstico; em 14,3\%, se passou um ano; em 7,1\%, se passaram dois anos entre a percepção dos pais de que havia algo errado com seu filho e o primeiro diagnóstico de autismo; em 21,4\%, se passaram três anos; e em 7,1\% dos casos, se passaram quatro anos.

Já no que diz respeito às psicoses, em $33,3 \%$ dos casos se passou menos de um ano entre a percepção dos pais de que havia algo errado com seu filho e o primeiro diagnóstico de psicose; em $11,1 \%$ dos casos, um ano; em 22,3\%, três anos; em $22,2 \%$, seis anos ou mais.

Os métodos disponíveis para a detecção precoce do autismo permitem a identificação de traços da psicopatologia em questão em bebês a partir dos três meses de idade. Porém, os dados obtidos revelam o fato do diagnóstico formal, no caso do autismo, ser dado aos dois anos em $21,4 \%$ dos casos; aos três anos, em $14,3 \%$; aos quatro anos, em $28,6 \%$; com cinco anos, em $18,6 \%$; e aos seis anos, em $7,1 \%$ dos casos.

No caso das psicoses, os dados são ainda mais preocupantes. Em 5\% dos casos, o diagnóstico formal é dado com cinco anos; em 5\%, com seis anos; em $20 \%$, aos sete anos; em $70 \%$, com oito anos ou mais. Além disso, no caso das psicoses, $25 \%$ dos casos estudados ainda estavam por se esclarecer.

Os dados obtidos também revelam provável insegurança e falta de contato com os sinais indicativos de um diagnóstico precoce do autismo e das psicoses infantis por parte dos médicos da primeira infância. No caso do autismo, em $71,4 \%$ dos casos, os pacientes foram encaminhados ao Centro de Atenção Psicossocial Infantil por alguma instituição de saúde; para 14,3\% dos pacientes, o encaminhamento se fez por alguma instituição de ensino; e somente $14,3 \%$ dos pacientes foram encaminhados por algum profissional da saúde; no caso, psicólogos.

Em relação às psicoses, $75 \%$ dos pacientes foram encaminhados por alguma instituição de saúde; $20 \%$ deles, por alguma instituição de ensino; e somente em $5 \%$ dos casos os pacientes foram encaminhados por algum profissional da saúde (um neurologista).

Além da dificuldade na realização do diagnóstico do autismo e das psicoses infantis, o presente trabalho revela também uma dificuldade por parte de profissionais e instituições de saúde em promover um tratamento para estas crianças. Em se tratando de autismo, para $85,7 \%$ dos pacientes, o primeiro tratamento específico se deu no próprio Centro de Atenção Psicossocial Infantil. Nesses casos, 92,9\% dos pacientes já haviam passado por outras instituições, como Associação dos Pais e Amigos dos Excepcionais, Hospital Menino Jesus, Sociedade Pestalozzi de São Paulo, Associação de Amigos do Autista e Central de Atendimento Especializado em Consultas Médicas, antes de chegar ao Centro de 
Atenção Psicossocial Infantil. Não há nos prontuários, entretanto, qualquer especificação, pelos pais, do tratamento eventualmente recebido pela criança nessas instituições.

Já no que diz respeito às psicoses infantis, para $70 \%$ dos casos, o primeiro tratamento específico se deu no Centro de Atenção Psicossocial Infantil; para 20\%, em alguma instituição de saúde (Hospitais e Unidades Básicas de Saúde); e para $10 \%$, por algum profissional da saúde.

Os dados apresentados revelam, ainda, uma sobrecarga de trabalho nos Centros de Atenção Psicossocial Infantil do município de São Paulo. A Diretora da instituição parceira revela a incapacidade em atender toda a demanda existente. Em alguns momentos, há até mesmo fila de espera para o acolhimento do paciente pela instituição e o consequente início do atendimento.

\section{Considerações finais}

Com base nos dados desta pesquisa, é possível estabelecer algumas diretrizes de encaminhamento a serem adotadas, para que o tratamento do autismo e da psicose possam se dar de maneira cada vez mais precoce e efetiva.

Fica evidente a necessidade de se trabalhar, na clínica do autismo e das psicoses infantis, com os pais das crianças. Os relatos e as suspeitas desses pais devem ser investigados e utilizados de maneira a auxiliar os profissionais da saúde em seu diagnóstico, isto porque os mesmos revelam-se capazes de uma percepção inicial da patologia anterior à dos profissionais e instituições de saúde. Nos casos das psicoses, ao lado do trabalho proposto acima, será preciso entender por que a percepção dos pais de sinais geradores de preocupação se dá com uma frequência reduzida e de forma tardia, em relação aos casos de autismo. Afinal, esse dado poderia implicar um menor comprometimento dos pais para com o tratamento de seus filhos.

Para evitar os descaminhos - passagem frustrada por profissionais e instituições de saúde - entre a percepção de sinais geradores de preocupação, por parte dos pais, e o primeiro diagnóstico formal da patologia, seguido do início do tratamento, será preciso trabalhar junto aos profissionais e instituições de saúde mais procurados ${ }^{4}$ por esses pais. Só assim o diagnóstico do autismo ou das

4. Os profissionais mais procurados são os neurologistas, pediatras; psicólogos e fonoaudiólogos; psiquiatras e otorrinolaringologistas; neuropediatras e oftalmologistas. Quanto às instituições: Hospital das Clínicas, Associação dos Pais e Amigos dos Excepcionais; Hospital Infanto-Ju- 
psicoses, e consequentemente o início de um tratamento, podem ocorrer o mais rápido possível. Trata-se, portanto, de criar um espaço de diálogo entre tais profissionais e instituições de saúde, de um lado, e os locais de tratamento especializado ao autista e ao psicótico. Assim, seria necessário disseminar, junto a esses profissionais e instituições, conhecimentos relativos à detecção precoce de patologias graves, colaborando, portanto, para o desenvolvimento da segurança e autonomia nos encaminhamentos a atendimentos especializados.

A falta de conhecimento e de autonomia, por parte de profissionais da saúde, em relação ao diagnóstico e o encaminhamento nos casos de autismo e psicose infantil, também pode ser percebido pelo fato de somente $14,3 \%$ dos pacientes, nos casos de autismo, e 5\% dos pacientes psicóticos, terem sido encaminhados à instituição parceira por profissionais da área. Esse dado revela que o conhecimento sobre o autismo e a psicose, assim como a habilidade de diagnóstico e o futuro encaminhamento, estão concentrados em instituições, ${ }^{5} \mathrm{o}$ que indica a necessidade de investimento na capacitação de profissionais da saúde, principalmente médicos da primeira infância.

No caso das psicoses, além da necessidade de agilizar a realização do diagnóstico da patologia em questão e do encaminhamento dos pacientes a um tratamento, os dados obtidos apontam a necessidade de pensarmos qual seria a concepção de psicose infantil dos profissionais do Centro de Atenção Psicossocial Infantil estudado, quais as dificuldades que os mesmos encontram para fechar um diagnóstico, e quais as implicações destas dificuldades nos tratamentos. Este diagnóstico se mostrou mais difícil e gerador de maior insegurança entre os profissionais em comparação ao diagnóstico de autismo. Assim, além de o diagnóstico da psicose infantil ocorrer de forma mais tardia que o do autismo, em $25 \%$ dos casos de psicose estudados o diagnóstico ainda estava por se esclarecer, enquanto que em $100 \%$ dos casos de autismo o diagnóstico já se encontrava fechado.

Além da necessidade de minimizar descaminhos e de agilizar caminhos para que o diagnóstico e o tratamento tanto do autismo quanto das psicoses se deem

venil Cândido Fontoura; Unidades Básicas de Saúde; Sociedade Pestalozzi de São Paulo, Associação de Amigos do Autista e Ambulatórios de Especialidades; Hospital Menino Jesus, Escola Paulista de Medicina, Central de Atendimento Especializado em Consultas Médicas e Hospital do Servidor Público Municipal.

5. Unidades Básicas de Saúde, Hospital Infanto-Juvenil Cândido Fontoura, Associação dos Pais e Amigos dos Excepcionais, Ambulatórios de Especialidades; Hospital das Clínicas, Hospital Menino Jesus, Sociedade Pestalozzi de São Paulo, Escola Paulista de Medicina, Hospital do Servidor Público Municipal e Associação de Amigos do Autista. 
de maneira cada vez menos tardia, a pesquisa revelou a não efetivação, por parte dos médicos da primeira infância, de diagnósticos precoces de autismo ou de psicoses. O que aponta para a consequente necessidade de difundir-se, no meio médico, por meio de capacitações, o conhecimento disponível.

Os médicos da primeira infância são parceiros indispensáveis, tanto no sentido de viabilizar o tratamento do autismo e das psicoses, de forma progressivamente precoce, quanto para a redução dos descaminhos existentes no percurso de famílias de crianças autistas e psicóticas. Convém lembrar, portanto, o fato desses profissionais possuírem acesso privilegiado à dupla mãe/bebê desde os primeiros meses de vida.

Na França, Marie-Christine Laznik desenvolve um trabalho de formação de médicos do serviço público e um projeto de pesquisa para o reconhecimento deste trabalho pelo governo, de modo a tornar a consulta pediátrica obrigatória na França (entre os quatro e os nove meses) também um espaço para a avaliação do laço Outro primordial/bebê. No Brasil já existem iniciativas, como a do grupo de pesquisa desse projeto, coordenado pela profa. dra. Silvana Rabello.

Além disso, os dados obtidos sugerem a necessidade, frente às psicoses infantis e ao autismo, dos profissionais e das instituições de saúde redobrarem sua atenção, em casos de pacientes provenientes de famílias desestruturadas. Em relação às psicoses infantis, deve-se, também, redobrar a atenção frente a famílias com histórico de problemas de saúde mental.

A título de conclusão, esta pesquisa pode contribuir com uma reflexão acerca de caminhos a serem percorridos, objetivando possibilitar a atuação de profissionais da saúde não especializados em autismo ou psicoses infantis, no sentido de abreviar a trajetória de seus pacientes rumo a um atendimento especializado. Ação de fundamental importância para viabilizar as intervenções clínicas em quadros ainda não cronificados.

Agradecimentos: Agradecemos a todos os membros do grupo maior de pesquisa e à Maria Clara de Araújo Fernandes.

\section{Referências}

LAZNIK, M-C. Rumo à palavra: três crianças autistas em psicanálise. Tradução Monica Seincman. São Paulo: Escuta, 1997.

Poderia a teoria lacaniana da pulsão fazer avançar a pesquisa sobre o autismo? Psicanálise e clínica de bebês, Curitiba, n. 4, p. 76-90, 2000. 


\section{ARTIGOS}

Interações sonoras entre bebês que se tornaram autistas e seus pais. In:

BeRnARDino, L.M.F. (Org.). O que a psicanálise pode ensinar sobre a criança, sujeito em constituição. São Paulo: Escuta, 2006. p. 95-106.

. A voz da sereia: o autismo e os impasses da constituição do sujeito. Salvador: Ágalma, 2004.

. Entre estereotipia e linguagem. In: WANDERLEy, D.B. (Org.). A voz da sereia: o autismo e os impasses na constituição do sujeito. Salvador: Ágalma, 2004. p. 132-151.

\section{Resumos}

(Considerations on early diagnosis in clinical work with autistic and psychotic children)

This article is about an academic study based on Marie-Christine Laznik's theory. The purpose is to investigate, through the study of patient's files, the way infantile autism and psychosis were diagnosed in a mental health institution. The study also investigated the children's family histories before arriving at the institution. The results show that the treatment of autistic and psychotic children begins as a very late age. Health professionals and institutions fail to note the signs for early detection and take long periods to provide diagnoses and proposed adequate treatment procedures. In addition, most health professionals seem unprepared to deal with these psychopathologies. This study revealed measures that can be taken to allow treatment of autistic and psychotic children to begin earlier.

Key words: Autism, child, early diagnosis, psychosis, mental health

(Considérations sur le diagnostic précoce dans la clinique de l'autisme et des psychoses infantiles)

Cet article décrit une recherche d'initiation scientifique basée sur la théorie de Marie Christine Laznik. Nous avons analysé des dossiers médicaux d'un Centre de Soins Psychosociaux pour Enfants de la ville de São Paulo pour étudier l'émission des diagnostics précoces de l'autisme et des psychoses infantile et pour retracer les histoires de ces familles jusqu'à leur arrivée à cette institution. Notre recherche montre que les enfants autistes et psychotiques sont traités de façon tardive dû au manque d'un dépistage précoce, dû aux trop longs délais des diagnostics formels et de la prise en charge des patients, ainsi qu'à l'insécurité des professionnels de santé par rapport à ces graves psychopathologies. Les résultats de cette recherche pourraient contribuer à élaborer de futures actions et les besoins qui exigent plus d'attention pour que les traitements de l'autisme et des psychoses infantiles puissent être mis en place plus tôt en ce qui concerne les tableaux cliniques non chronicisés.

Mots clés: Autisme infantile, enfant, diagnostique précoce, psychoses, santé mentale 
(Consideraciones sobre el diagnóstico precoz en la clínica del autismo y de las psicosis infantiles)

Este artículo tiene como referencia una investigación realizada como iniciación científica y se basó en la teoría de Marie-Chistine Laznik. El trabajo consistió en estudiar los historiales clínicos de niños autistas y sicóticos tratados en un centro de salud mental (Centro de Atención Psicosocial Infantil) en el municipio de São Paulo. Se objetivava comprender como se procesó el diagnóstico precoz del autismo y de la psicosis infantiles y cual fue la trayectoria de esas familias hasta su llegada a esos centros de salud mental. La investigación reveló que el inicio del tratamiento de niños con autismo y psicosis infantil comienza tarde porque no se hace una detección precoz de esas condiciones, los profesionales y las instituciones de salud mental demoran para hacer un diagnóstico formal y para derivarlos para tratamiento y, por la inseguridad de los profesionales de salud para lidiar con estas psicopatologías graves. Los resultados de esta investigación ayudan a pensar acciones futuras, apuntan para la necesidad de una concentración mayor de esfuerzos para que el tratamiento del autismo y de las psicosis infantiles pueda acontecer más tempranamente en vista de ser cuadros aún no cronificados.

Palabras clave: Autismo infantil, niñez, diagnóstico precoz, psicosis, salud mental

(Erwägungen zur frühzeitigen Diagnose in der Behandlung von Autismus und der Kinderpsychosen).

In diesem Beitrag wird anhand der Auswertung von Patientenkarteien untersucht, wie es zur frühzeitigen Diagnose von Autismus und der Kinderpsychosen in einem Behandlungszentrum für Kinderpsychosen von São Paulo kam und welchen Weg diese Familien zurückgelegt haben bis sie zur Einrichtung gelangten. Die Untersuchung ergab, dass die Behandlung von autistischen und psychotischen Kindern sehr spät beginnt, weil keine frühzeitige Diagnose gestellt wird, weil die Spezialisten und spezialisierten Gesundheitseinrichtungen zu lange brauchen, um eine formale Diagnose $z u$ stellen und um den Patienten zur angemessenen Behandlung weiterzuleiten, wegen der Unsicherheit der Berufstätigen im Gesundheitswesen im Umgang mit diesen schwierigen Psychopathologien. Die Ergebnisse der Untersuchung tragen dazu bei, zukünftige Initiativen zu entwickeln und Bereiche zu erkennen, die mehr Aufmerksamkeit erfordern, um die Behandlung von Autismus und von Kinderpsychosen frühzeitiger einzuleiten, solange das Krankheitsbild noch nicht chronisch ist.

Schlüsselwörter: Autismus von Kindern, frühzeitige Diagnose, Psychosen, geistige Gesundheit 

EM TABELAS FORMULADAS POR DESCRITORES

\begin{tabular}{|c|c|}
\hline \multicolumn{2}{|c|}{$\begin{array}{c}1 \text { - PAIS QUE PERCEBEM ALGUMA COISA DE } \\
\text { ERRADO COM SEU FILHO }(\%)\end{array}$} \\
\hline AUTISMO & PSICOSE \\
\hline 78,6 & 45 \\
\hline
\end{tabular}

2 - IDADE NA QUAL OS PAIS PERCEBEM ALGUMA COISA DE ERRADO COM SEU FILHO (\%)

\begin{tabular}{|c|c|c|c|c|c|c|c|c|c|}
\hline IDADE (ANOS) & $1-$ & 2 & 3 & 4 & 5 & 6 & 7 & $8+$ & INDISPONIVEL \\
\hline AUTISMO & 36,4 & 27,3 & 18,2 & & & & & & 18,1 \\
\hline PSICOSE & & 11,1 & & 22,35 & & & 22,35 & 33,3 & 10,9 \\
\hline
\end{tabular}

\begin{tabular}{|c|c|c|c|c|c|c|c|c|c|}
\hline \multicolumn{8}{|c|}{3 - IDADE EM QUE E DADO UM DIAGNOSTICO FORMAL (\%) } \\
\hline IDADE (ANOS) & 1 & 2 & 3 & 4 & 5 & 6 & 7 & $8+$ & INDISPONIVEL \\
\hline AUTISMO & & 21,4 & 14,3 & 28,6 & 18,6 & 7,1 & & & 10 \\
\hline PSICOSE & & & & & 5 & 5 & 20 & 70 & \\
\hline
\end{tabular}

4-TEMPO ENTRE A PERCEPCCAO DOS PAIS E O DIAGNOSTICO FORMAL (\%)
\begin{tabular}{|c|c|c|c|c|c|c|c|c|}
\hline \multicolumn{1}{|c|}{ - TADE (ANOS) } & 1 & 2 & 3 & 4 & 5 & $6+$ & MENOS DE 1 & INDISPONIVEL \\
\hline IDAUTISMO & 14,3 & 7,1 & 21,4 & 7,1 & & & 14,3 & 35,8 \\
\hline PSICOSE & 11,1 & & 22,3 & & & 22,2 & 33,3 & 11,1 \\
\hline
\end{tabular}

\begin{tabular}{|c|c|c|c|c|c|c|c|c|c|c|}
\hline \multicolumn{2}{|c|}{5 -IDADE DE INICIO DO PRIMEIRO TRATAMENTO (\%) } \\
\hline IDADE (ANOS) & 1 & 2 & 3 & 4 & 5 & 6 & 7 & 8 & 9 & $10+$ \\
\hline AUTISMO & & 7,1 & 7,1 & 50,1 & 21,5 & 7,1 & 7,1 & & & \\
\hline PSICOSE & & & & & 15 & & 10 & & 5 & 70 \\
\hline
\end{tabular}

\begin{tabular}{|c|c|c|c|c|c|c|c|c|c|c|}
\hline \multicolumn{8}{|c|}{6 - IDADE DE INICIO DO TRATAMENTO NO CAPSi (\%) } \\
\hline IDADE (ANOS) & 1 & 2 & 3 & 4 & 5 & 6 & 7 & 8 & 9 & $70+$ \\
\hline AUTISMO & & & & 57,1 & 28,6 & 14,3 & & & & \\
\hline PSICOSE & & & & & 5 & 5 & 15 & & & 75 \\
\hline
\end{tabular}

\begin{tabular}{|c|c|c|c|c|c|c|c|c|}
\hline 7 - TEMPO QUE SE PASSOU ENTRE O PRIMEERO PROFISSIONALINSTITUCCAO CONSULTADO ATE A CHEGADA AO CAPSI (\%) \\
\hline ANOS & 1 & 2 & 3 & 4 & 5 & 6 & MENOS DE 1 & INDISPONIVEL \\
\hline AUTISMO & 28,6 & 21,4 & 14,3 & 14,3 & & & 21,4 & \\
\hline PSICOSE & 20 & 5 & & & 10 & 10 & 50 & 5 \\
\hline
\end{tabular}

\begin{tabular}{|c|c|c|c|c|c|c|c|c|c|}
\hline 8-NUMERO DE PROFISSIONALIINSTITUICAO PROCURADOS ATE SER DADO UM DIAGNOSTICO FORMAL \\
\hline$\#$ & 1 & 2 & 3 & 4 & 5 & 6 & 7 & $8+$ & INDISPONIVEL \\
\hline AUTISMO & 28,6 & 14,3 & 7,1 & 28,6 & 7,1 & & & & 14,3 \\
\hline PSICOSE & 60 & 30 & 5 & 5 & & & & & \\
\hline
\end{tabular}

\begin{tabular}{|c|c|c|}
\hline \multicolumn{3}{|c|}{$\begin{array}{l}\text { 9 - COMO SE DEU O ENCAMINHAMENTO AO } \\
\text { CAPSi (\%) }\end{array}$} \\
\hline & AUTISMO & PSICOSE \\
\hline $\begin{array}{l}\text { INSTITUICAOA } \\
\text { DE SAÚDE }\end{array}$ & 71,4 & 75 \\
\hline $\begin{array}{l}\text { PROFISSIONAL } \\
\text { DA SAÚDE }\end{array}$ & 14,3 & 5 \\
\hline $\begin{array}{l}\text { INSTITUICAAO } \\
\text { DE ENSINO }\end{array}$ & 14,3 & 20 \\
\hline
\end{tabular}

\begin{tabular}{|c|c|}
\hline \multicolumn{2}{|c|}{$\begin{array}{l}\text { 10 - CAPSI COMO PRIMEIRO LUGAR DE } \\
\text { TRATAMENTO }(\%)\end{array}$} \\
\hline AUTISMO & PSICOSE \\
\hline 85,7 & 70 \\
\hline \multicolumn{2}{|c|}{ 11-DIAGNOSTICOS NAO ESCLARECIDOS (\% } \\
\hline AUTISMO & PSICOSE \\
\hline 0 & 25 \\
\hline
\end{tabular}

\begin{tabular}{|c|c|}
\hline \multicolumn{2}{|c|}{$\begin{array}{l}\text { 72-CASOS LIGADOSA SAUDE METAL NA } \\
\text { FAMILIA (\%) }\end{array}$} \\
\hline AUTISMO & PSICOSE \\
\hline 7,1 & 30 \\
\hline
\end{tabular}

13 - RELAÇÅO DO DIAGNÓSTICO COM A VIVÉNCIA EM UMA FAMILIA DESESTRUTURADA (\%) \begin{tabular}{|l|l}
\hline AUTISMO & PSICOSE \\
\hline
\end{tabular} \begin{tabular}{l|l}
\hline 28,6 & 25 \\
\hline
\end{tabular} 


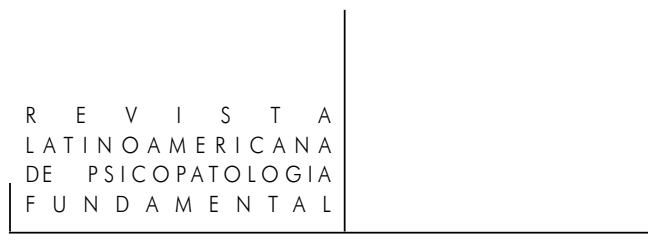

Citação/Citation: VISANI, P.; RABELLO, S. Considerações sobre o diagnóstico precoce na clínica do autismo e das psicoses infantis. Revista Latinoamericana de Psicopatologia Fundamental, São Paulo, v. 15, n. 2, p. 293-308, junho 2012.

Editor do artigo/Editor: Prof. Dr. Manoel Tosta Berlinck

Recebido/Received: 15.7.2011 / 7.15.2011 Aceito/Accepted: 25.10.2011 / 10.25.2011

Copyright: () 2009 Associação Universitária de Pesquisa em Psicopatologia Fundamental/ University Association for Research in Fundamental Psychopathology. Este é um artigo de livre acesso, que permite uso irrestrito, distribuição e reprodução em qualquer meio, desde que o autor e a fonte sejam citados / This is an open-access article, which permits unrestricted use, distribution, and reproduction in any medium, provided the original author and source are credited.

Financiamento/Funding: As autoras declaram não ter sido financiadas ou apoiadas / The authors have no support or funding to report.

Conflito de interesses/Conflict of interest: As autoras declaram que não há conflito de interesses / The authors declare that has no conflict of interest.

\section{Paola Visani}

Graduada em Psicologia pela Pontifícia Universidade Católica de São Paulo - PUC-SP (São Paulo, SP, Br); concluiu em 2009 um trabalho de Iniciação Científica intitulado Captação de bebês de risco - detecção precoce de psicopatologias graves, sob a orientação da profa. dra. Silvana Rabello, com bolsa do PIBIC-CEP. Concluiu em 2010 sua segunda pesquisa de Iniciação Científica, Captação de bebês de risco - detecção precoce de psicopatologias graves II, com a mesma orientadora, agora com bolsa PIBIC-CNPq.

Rua Caraíbas, 719/101 - Perdizes

05020-000 São Paulo, SP, Br

e-mail: paola_visani@hotmail.com

\section{Silvana Rabello}

Bacharel em Psicologia pela Pontifícia Universidade Católica de São Paulo - PUC-SP (São Paulo, SP, Br) (1982); mestrado em Educação - Distúrbios da Comunicação (1990) e doutorado em Psicologia (Psicologia Clínica) pela mesma Universidade (2004). Assistente Doutor da Pontifícia Universidade Católica de São Paulo - PUC-SP e membro da Associação Universitária de Pesquisa em Psicopatologia Fundamental (São Paulo, SP, Br). Coordena o Projeto Espaço Palavra (aprimoramento clínico institucional na área do autismo e psicose) e o curso de Pós-Graduação Lato Sensu em Teoria Psicanalítica, além de coordenar, há mais de dez anos um grupo de pesquisa no campo da Detecção Precoce de Psicopatologias Graves, há mais dez anos, envolvendo alunos em Iniciação Científica e aprimorando-os na área das psicoses - uma leitura psicanalítica. Tem experiência na área de Psicanálise, com ênfase em Psicopatologias Graves da Infância e as questões que envolvem a Constituição da Subjetividade, atuando principalmente nos seguintes temas: autismo e psicose infantil, clínica psicanalítica, constituição do sujeito e detecção precoce de psicopatologias graves. Faz parte da equipe da Clínica Interdisciplinar prof. dr. Mauro Spinelli e do Departamento de Saúde Mental da Sociedade de Pediatria de São Paulo.

Rua Dr. Homem de Mello, 736 - Perdizes

05414-000 São Paulo, SP, Br

e-mail: silvanarabb@hotmail.com 